\title{
Hotel characteristics and seasonality in prices: an analysis using Spanish tour operators' brochures
}

\author{
JOSEP-MARIA ESPINET \\ Departament d'Economia, Universitat de Girona, Facultat de Ciències Econòmiques i \\ Empresarials, Campus Montilivi, 17071 Girona, Spain. \\ E-mail: josepmaria.espinet@udg.edu.
}

\section{MODEST FLUVIÀ}

Departament d'Economia, Universitat de Girona, Facultat de Ciències Econòmiques i Empresarials, Campus Montilivi, 17071 Girona, Spain. E-mail: modest.fluvia@udg.edu.

\section{RICARD RIGALL-I-TORRENT}

Departament d'Economia, Universitat de Girona, Facultat de Ciències Econòmiques i Empresarials, Campus Montilivi, 17071 Girona, Spain. E-mail: ricard.rigall@udg.edu. (Corresponding author.)

\section{Albert SAlÓ}

ESADE Business School, Universitat Ramon Llull, Av Torre Blanca 59, Sant Cugat del Vallès, Barcelona, Spain, and Departament d'Economia, Universitat de Girona. E-mail: albert.salo@esade.edu.

Seasonality in the tourism sector has been a major concern for policy makers, managers and other stakeholders. Many studies have analysed seasonality from the point of view of the number of visitors. However, there appear to be no studies focusing on seasonality in prices and on how to smooth out seasonal patterns. This paper analyses how hotel characteristics affect seasonality in prices using brochure data on 1,776 hotels in 32 sun-and-beach destinations in 11 countries. The authors find that, after controlling for destinationspecific variables that may cause variations in prices through demand shifts (such as climatic conditions, exchange rates or marketing expenditures), more hotel services and higher star ratings are associated with fewer seasonal variations in hotel prices.

Keywords: seasonality; hotels; prices; destinations 
Compared to other industries, one of the differential characteristics of the tourism industry is its seasonality. Prices and occupation levels tend to fluctuate (sometimes wildly) throughout the year (see, for instance, Goh and Law, 2002; Koenig and Bischoff, 2003; Gil-Alana et al, 2004; Cuñado et al, 2005; Kulendran and Wong, 2005). For instance, in the case of sun-and-beach destinations, prices and occupation levels usually reach a peak during the summer period. Seasonality in the tourism sector has been a major concern for policy makers, managers and other stakeholders, since it is the source of many 'problems', such as short business operating seasons, underutilization of capital assets, short-term employment or maintaining service, and product quality standards in the absence of long-term employees (Baum and Lundtorp, 2001; Lundtorp et al, 2001).

Many studies have analysed seasonality in tourism (for a review, see Chung, 2009). These studies focus either on the demand side (Kennedy and Deegan, 2001; Lim and McAleer, 2001; Lundtorp et al, 2001; Koenig and Bischoff, 2003; Rosselló Nadal et al, 2004) or on the supply side (Krakover, 2000; Flognfeldt, 2001; Jeffrey and Barden, 2001; Capó Parrilla et al, 2007). Usually, it is assumed that there are eight main reasons for seasonality (Lundtorp et al, 2001): natural (associated with climate and seasons of the year); institutional (linked to cultural and religious factors); social pressure and fashion; a sporting season; inertia and tradition; business customs; calendar effects (number of weekends, official holidays); and supply constraints. Seasonality studies have focused their attention on the effects of these different types of seasonal patterns on destinations and tourism-related businesses (Butler, 2001; Kennedy and Deegan, 2001; Sorensen, 2001). Both the public and private sectors have devoted many efforts to overcome the problems due to seasonality by trying to stimulate demand during shoulder and off-season periods (Baum and Lundtorp, 2001; Commons and Page, 2001; Flognfeldt, 2001; Klemm and Rawel, 2001; Koenig and Bischoff, 2003; Jang, 2004; Fernández-Morales and MayorgaToledano, 2008).

In spite of the profusion of research on seasonality, as far as we know there are no studies analysing seasonality in prices. In spite of this neglect (due possibly to the difficulty of collecting prices for different destinations), analysing the seasonal patterns in prices is valuable for policy makers, managers and other stakeholders. Indeed, prices play (at least) two crucial roles in the tourism sector. On the one hand, price is a key variable for the marketing mix of tourism firms: a lot of research has been devoted to the analysis of prices as signals of quality (Wolinsky, 1983; Milgrom and Roberts, 1986; HjorthAndersen, 1991; Caves and Greene, 1996; Jones and Hudson, 1996) and to decomposing the prices of tourism goods and services into the elements that give satisfaction to consumers (Hartman, 1989; Aguiló et al, 2001; Papatheodorou, 2002; Cox and Vieth, 2003; Espinet et al, 2003; Monty and Skidmore, 2003; Haroutunian et al, 2005; Thrane, 2005; Rigall-I-Torrent and Fluvià, 2007; Falk, 2008; Rigall-I-Torrent and Fluvià, 2011). On the other hand, prices allow tourism firms to bring together demand and supply: this role of prices is especially important in the tourism sector, since it contributes to the generation of revenue for non-storable products in the presence of low variable costs and high (fixed) capacity costs (Kimes, 2000; Talluri and van Ryzin, 2004; Phillips, 2005; Shy, 2008). As a result of the two roles played by prices, tourism 
managers face a trade-off. Lower prices at the trough of the tourism season mean higher revenues (and profits) for firms and destinations, but they may also be perceived by some potential customers as a loss of quality and/or exclusivity, and the tourism product or destination may be regarded as less valuable. As a result of this second effect, the brand image of the product/destination may ultimately become seriously damaged and tourism firms' future revenues and profits may be put at risk. Consider, for instance, the notorious case of Salou, one of the most important tourism destinations in Spain, where low prices during the low season attract tourists who put in jeopardy the image of highquality tourism the destination wishes to promote (see, for instance, Balsells, 2010; Martin, 2010).

This paper analyses the factors that explain seasonal variations in prices for hotels in sun-and-beach destinations. In contrast to most articles on seasonality (which study specific geographical areas), this article takes an international perspective and considers seasonality in prices in sun-and-beach resorts in the Mediterranean, the Canary Islands, the Adriatic and the Caribbean, among other places, relying on Spanish tour operators' brochures. The paper starts by setting out the framework for the analysis of seasonality in prices. Next the data, the methodology employed and the main results are spelled out. After that, the paper's results are used to draw implications for the management of tourism firms and destinations. The paper ends with our conclusions.

\section{Seasonality in prices: framework of analysis}

\section{Sources of seasonal variations in prices}

As noted in the introduction, seasonality studies in tourism have focused their attention on the number of visitors arriving at different destinations or staying at different types of accommodation. Capó Parrilla et al (2007) analyse the accommodation determinants of seasonal patterns in the Balearic Islands (Spain) and find that establishments offering a higher level of service, measured in terms of star category, tend to have a longer opening period during the year. In a similar vein, the present paper aims to elucidate empirically how hotel characteristics affect seasonality in prices.

To do this, it is important to reflect on the relationship between seasonality in price and seasonality in the number of visitors. The absence of observed seasonality in prices does not imply that hotel occupation levels do not show seasonal patterns. Conversely, if seasonality in prices is observed, it is not possible to conclude that there is no seasonality in hotel occupation levels. Indeed, elementary economic theory tells us that, under perfect competition, market prices are the outcome of the interaction between demand and supply. At a given market price, individual firms will produce a positive quantity of output whenever the market price is higher than their average variable cost, since firms would then be earning sufficient revenue to pay all their variable costs. Otherwise, firms would achieve higher profits by shutting down. In this setting, the seasonality in prices observed in tourism markets can be easily explained in terms of changes in demand and supply. At the peak of the tourism period (for instance, in the summer months in the case of Mediterranean 
destinations) demand is clearly higher than in trough periods. If supply remains constant, this implies that market prices will rise to match supply and demand. Nevertheless, some tourism businesses that remain closed during the trough period because market prices make their activity unprofitable may find it worthwhile to open their premises in the peak period. This effect can be understood as shifting supply to the right. (Obviously, since building new tourism infrastructure takes time, the possibilities of increasing supply are limited in the short run.) As will be apparent, the final outcome in terms of the market price depends on the relative magnitude of the demand and supply shocks.

This elementary informal analysis shows that there are three variables to take into account when analysing seasonality: demand, supply and price. The last brings together demand and supply. This implies that, when the market price changes throughout the tourism season, this can be the result of a shift in demand, a shift in supply or both. Besides, even if no changes in the market price are observed, it is possible that both demand and supply have shifted. Nevertheless, when one considers the period of the year when all hotels are opened, supply can be assumed to be fixed and changes in prices will be due solely to shifts in demand. With respect to the latter, it is usually assumed (see, for instance, Crouch, 1994) that, in addition to own price, tourism demand depends on income, exchange rates, transportation costs and marketing expenditure. Furthermore, Capó Parrilla et al (2007) find that, from a psychographic perspective, off-season tourists are motivated by factors other than beaches and the weather and that higher numbers of hotel characteristics and services are associated with lower fluctuations in demand. Finally, it is important to keep in mind that this framework of analysis considers a perfect competition setting. Under monopolistic competition or oligopoly, it is important to consider the strategic behaviour of firms when setting prices.

Given these considerations, in order to analyse how hotel characteristics may explain seasonal variations in prices, we establish and estimate a relationship such as:

$$
P S_{b}=f\left(\mathbf{c}_{b}, l_{b}, t_{b} ; \boldsymbol{\beta} ; \varepsilon\right)
$$

where $P S_{b}$ is a measure of the seasonality in prices for hotel $h, \mathbf{c}_{b}$ is a vector of hotel characteristics (such as star rating and services available, accommodation type or whether the hotel is part of a chain), $l_{b}$ refers to the destination where the hotel is located, $t_{b}$ is the tour operator which sells hotel $b$ 's rooms (a variable that controls for differences in marketing strategies between tour operators), $\boldsymbol{\beta}$ is a vector of parameters which show the effect of each location and characteristics on seasonality in prices; and $\varepsilon$ is an i.i.d. random disturbance which captures non-systematic events. Notice that the destination implicitly captures, among other things, the effects of differentials in exchange rates, transportation costs, marketing expenditure and climatic factors. If a short period is considered (say, a year), it seems reasonable to assume that neither consumers' income nor preferences change. Therefore, assuming that supply is fixed, Equation (1) would capture all the relevant sources of seasonal variations in prices. Although we do not have data regarding the degree of competition in the different destinations analysed in this paper, all have a number of hotels 
with a rating high enough to preclude (at least in theory) strategic behaviour when setting prices.

\section{Measuring seasonal variations in prices}

There are many possible approaches to analysing the determinants of seasonality in hotel prices. Nevertheless, any approach must start by defining some measure of seasonality. The literature on tourism seasonality typically uses three ways to measure the distribution of visitors (see Lundtorp, 2001, for an in-depth review of the measures used): the coefficient of seasonal variation, the seasonality ratio and the Gini coefficient. The coefficient of seasonal variation is computed as the standard deviation of the number of visitors in the different units of time (say months, for instance) divided by the average number of visitors per unit of time. The seasonality ratio is the result of dividing the highest number of visitors in a period over the average number of visitors. Finally, the Gini coefficient is defined as:

$$
G=\frac{2}{n}\left(\sum_{i=1}^{n} i f_{i}-\frac{n+1}{2}\right),
$$

where, considering months as the time period, $n$ is the number of months, and $f_{i}$ is defined as $f_{i} \equiv v_{i} / v_{0}$, where $v_{i}$ is the number of visitors in month $i$, and $v_{0}$ is the total number of visitors during the year.

When analysing seasonality in prices, the Gini coefficient does not seem appropriate, since there is no meaningful price measure equivalent to the total number of visitors. The other measures of seasonality in the case of visitors can easily be adapted to the analysis of prices. The coefficient of variation of the prices of a hotel $b$ can be defined as:

$$
C V p_{b} \equiv \frac{\sqrt{\frac{1}{N} \sum_{n=1}^{N}\left(p_{n, b}-\frac{1}{N} \sum_{n=1}^{N} p_{n, b}\right)^{2}}}{\frac{1}{N} \sum_{n=1}^{N} p_{n, b}},
$$

where $p$ is the price and $n=1, \ldots, N$ is the month of the year. This index has a lower bound of 0 when there is no seasonality and no upper bound when seasonality exists. The seasonality ratio of the prices of a hotel $b$ is defined as:

$$
S R p_{b} \equiv \frac{\max \left\{p_{1 b}, \ldots, p_{N b}\right\}}{\frac{1}{N} \sum_{n=1}^{N} p_{n, b}},
$$

which again has a lower bound of 1 when there is no seasonality and no upper bound. An additional measure of seasonality in prices might consider the maximum price charged in a year divided by the minimum price; that is,

$$
S p_{b} \equiv \frac{\max \left\{p_{1 b}, \ldots, p_{N b}\right\}}{\min \left\{p_{1, b}, \ldots, p_{N b}\right\}},
$$


which has a lower bound of 1 when there is no seasonality and no upper bound. Although other measures of seasonality in prices are possible (for instance, the difference between maximum and minimum prices in absolute values), measures (3), (4) and (5) have the advantage of being unit-free.

\section{Methodology and results}

\section{Data}

This study relies on hotel prices drawn from Spanish tour operators' brochures for 2002. The tour operators considered are those with the highest market shares in Spain: Iberojet, Rhodasol, Marsol, Mundicolor, Solplan, Vivatours, Travelplan and El Corte Inglés. All the hotels listed by these tour operators in 32 different tourism destinations (in 11 countries in the Mediterranean, the Atlantic and the Caribbean) are included in the database (see Table 1). This amounts to 1,776 hotels and 27,231 prices. The prices considered include the period from May to October, which is when most Spaniards take their vacations and also when the vast majority of the hotels in all the destinations analysed are open. This allows us to assume that supply is fixed, so that variations in prices due to demand fluctuations are captured by Equation (1). (Although in theory it is possible that hotels are open but their capacity is not fixed, we do not have data to contrast this hypothesis.) Specifically, the following dates are analysed: 10-17 May; 21-28 June; 5-12 July; 2-9 August; 6-13 September; 11-18 October. The prices used in this paper take into account the discounts specified in the brochures. Although it is not possible to consider other discounts on list prices (last-minute or based on age or club membership, for instance), it is reasonable to assume that brochure prices reflect 'expected' prices paid by tourists; that is, subject to deviations around the expected value (RigallI-Torrent and Fluvià, 2011). For the prices to be comparable, the price of transport and tours at the destination is not included in the prices analysed.

For each hotel the database also includes the relevant services as determined by Espinet et al (2003) and later confirmed by other authors (Haroutunian et al, 2005; Thrane, 2005; Rigall-I-Torrent and Fluvià, 2007, 2011), as well as variables describing the hotel star rating, accommodation type and whether the hotel is part of a chain or if it can be classified as an apartment hotel. A list of the variables included in the database and some descriptive statistics are shown in Table 2.

Figure 1 shows the monthly average prices for all the destinations analysed. From Figure 1 it is apparent that a clear seasonal pattern emerges for most destinations. Nevertheless, the ultimate sources of such patterns remain hidden.

\section{Model specification and estimation}

As noted in the previous section, the aim of this paper is to elucidate how hotel characteristics can explain seasonal variations in prices. Since, as far as we know, there are no previous studies on this specific topic, the literature does not offer guidance as to what empirical methodology to use. Nevertheless, following the mainstream tradition in economic research, this paper uses regression analysis to isolate the effects of the different relevant accommodation variables on price 
Table 1. Geographical distribution of the hotels analysed.

\begin{tabular}{|c|c|c|c|c|}
\hline Destination & Country & $\begin{array}{l}\text { Mainland/ } \\
\text { Island }\end{array}$ & Ocean/Sea & $\begin{array}{l}\text { Hotels } \\
\text { in the } \\
\text { sample }\end{array}$ \\
\hline Algarve & Portugal & Mainland & Atlantic & 47 \\
\hline Corfu & Greece & Island & Mediterranean (Ionic) & 11 \\
\hline Costa Blanca & Spain & Mainland & Mediterranean & 109 \\
\hline Costa Brava & Spain & Mainland & Mediterranean & 144 \\
\hline Costa Daurada & Spain & Mainland & Mediterranean & 86 \\
\hline Costa del Maresme & Spain & Mainland & Mediterranean & 68 \\
\hline Costa del Sol & Spain & Mainland & Mediterranean & 129 \\
\hline Crete & Greece & Island & Mediterranean & 32 \\
\hline Croatia & Croatia & Mainland & Mediterranean (Adriatic) & 17 \\
\hline Cuba & Cuba & Island & Caribbean & 86 \\
\hline Dominican Republic & Dominican Republic & Island & Caribbean & 70 \\
\hline French Riviera & France & Mainland & Mediterranean & 17 \\
\hline Fuerteventura & Spain & Canary Islands & Atlantic & 32 \\
\hline Gran Canaria & Spain & Canary Islands & Atlantic & 62 \\
\hline Ibiza & Spain & Balearic Islands & Mediterranean & 58 \\
\hline Kos & Greece & Island & Mediterranean (Aegean) & 7 \\
\hline Lanzarote & Spain & Canary Islands & Atlantic & 35 \\
\hline Madeira & Portugal & Island & Atlantic & 41 \\
\hline Majorca & Spain & Balearic Islands & Mediterranean & 219 \\
\hline Malta & Malta & Island & Mediterranean & 24 \\
\hline Menorca & Spain & Balearic Islands & Mediterranean & 38 \\
\hline Mexico & Mexico & Mainland & Caribbean & 98 \\
\hline Mykonos & Greece & Island & Mediterranean (Aegean) & 31 \\
\hline Naples & Italy & Mainland & Mediterranean & 9 \\
\hline Naxos & Greece & Island & Mediterranean (Aegean) & 5 \\
\hline Paros & Greece & Island & Mediterranean (Aegean) & 7 \\
\hline Rhodes & Greece & Island & Mediterranean (Aegean) & 14 \\
\hline Santorini & Greece & Island & Mediterranean (Aegean) & 29 \\
\hline Sicily & Italy & Island & Mediterranean & 64 \\
\hline Skiathos & Greece & Island & Mediterranean (Aegean) & 3 \\
\hline Tenerife & Spain & Canary Islands & Atlantic & 109 \\
\hline Tunisia & Tunisia & Mainland & Mediterranean & 75 \\
\hline
\end{tabular}

seasonality; that is, to conduct an analysis under the clause ceteris paribus. Thus, to identify the effect of the different variables on the seasonality of prices, the paper estimates different specifications of Equation (1) by OLS. (Notice that this way of proceeding is equivalent to the multivariate analysis of variance.)

Specifically, the paper uses the three different approaches described by (3), (4) and (5) to capture seasonality in prices. Notice that, since all the available dependent variables are dichotomic, the number of easily interpretable alternative specifications is clearly limited. Besides, introducing multiplicative interactions would clutter the model unnecessarily. Therefore, for each approach we consider the dependent variable in levels and in logs, so that we end up 
Table 2. Variables included in the database.

Type of variable Variable

Price

Services

available

at the hotel

Hotel star

rating

Accommodation
type

type

Hotel type

Chain or independent

Region

Hotel

Chain
Price per night

Hotel located in front of beach

Room services available

Garden or balcony available

Swimming pool available

Outdoor sport facilities available

Car park available

Animation activities available

Indoor sport facilities available

Hotel has been refurbished

1-star hotel

2-star hotel

3-star hotel

4-star hotel

5-star hotel

Lunch included

Breakfast included

Half board

Full board

All inclusive

Apartment hotel

Independent

Costa Brava (Spain)

Costa del Maresme (Spain)

Costa Daurada (Spain)

Costa Blanca (Spain)

Costa del Sol (Spain)

Ibiza (Spain)

Majorca (Spain)

Menorca (Spain)

Gran Canaria (Spain)

Fuerteventura (Spain)

Lanzarote (Spain)

Tenerife (Spain)

Sicily (Italy)

Corfu (Greece)

Dominican Republic

Mexico

Cuba

Tunisia

Malta

French Riviera (France)

Croatia
Mean

Standard Maximum Minimum deviation

$\begin{array}{llll}55.93846 & 28.39111 & 248.75 & 10.33333\end{array}$

0.4052943

$\begin{array}{lll}- & 1 & 0\end{array}$

0.9115909

0.9182729

0.9308661

0.5145207

0.6255461

0.5443331

0.5911077

0.4322796

0.0097661

0.0449756

0.4104343

0.4081213

0.1267026

0.0148533

0.078696

0.8135179

0.0159175

0.0770153

0.0583398

0.9416602

0.459779

0.540221

0.0981753

0.0539707

0.074531

0.0920072

0.0400925

0.0305834

0.0961192

0.0236443

0.0393215

0.0192753

0.0328964

0.074017

0.0205603

0.003598

0.0274994

0.0444616

0.0403495

0.0282704

0.0064251

0.0048831

0.0043691

0

$\begin{array}{ll}- & 1\end{array}$

0

0

0

$\begin{array}{ll}- & 1\end{array}$

$\begin{array}{ll}- & 1\end{array}$

$\begin{array}{ll}- & 1\end{array}$

0

0

$\begin{array}{lll}- & 1 & 0\end{array}$

$\begin{array}{lll}- & 1 & 0\end{array}$

$\begin{array}{lll}- & 1 & 0\end{array}$

$\begin{array}{lll}- & 1 & 0\end{array}$

$\begin{array}{lll}- & 1 & 0\end{array}$

$\begin{array}{lll}- & 1 & 0\end{array}$

$\begin{array}{lll}- & 1 & 0\end{array}$

$\begin{array}{lll}- & 1 & 0\end{array}$

$\begin{array}{lll}- & 1 & 0\end{array}$

$\begin{array}{lll}- & 1 & 0\end{array}$

$\begin{array}{lll}- & 1 & 0\end{array}$

$\begin{array}{lll}- & 1 & 0\end{array}$

$\begin{array}{lll}- & 1 & 0\end{array}$

$\begin{array}{lll}- & 1 & 0\end{array}$

$\begin{array}{lll}- & 1 & 0\end{array}$

$\begin{array}{ll}- & 1\end{array}$

$\begin{array}{lll}- & 1 & 0\end{array}$

$\begin{array}{lll}- & 1 & 0\end{array}$

$\begin{array}{lll}- & 1 & 0\end{array}$

$\begin{array}{lll}- & 1 & 0\end{array}$

$\begin{array}{lll}- & 1 & 0\end{array}$

$\begin{array}{lll}- & 1 & 0\end{array}$

$\begin{array}{lll}- & 1 & 0\end{array}$

$\begin{array}{lll}- & 1 & 0\end{array}$

$\begin{array}{lll}- & 1 & 0\end{array}$

$\begin{array}{lll}- & 1 & 0\end{array}$

$\begin{array}{lll}- & 1 & 0\end{array}$

$\begin{array}{lll}- & 1 & 0\end{array}$

$\begin{array}{lll}- & 1 & 0\end{array}$

$\begin{array}{ll}- & 1 \\ - & 1\end{array}$

$\begin{array}{lll}- & 1 & 0\end{array}$

$\begin{array}{lll}- & 1 & 0\end{array}$

$\begin{array}{lll}- & 1 & 0\end{array}$

$\begin{array}{lll}- & 1 & 0\end{array}$

$\begin{array}{lll}- & 1 & 0\end{array}$

$\begin{array}{lll}- & 1 & 0\end{array}$

$\begin{array}{lll}- & 1 & 0 \\ - & 1 & 0\end{array}$

$\begin{array}{lll}- & 1 & 0 \\ - & 1 & 0\end{array}$


Table 2 continued.

Type of variable Variable

\begin{tabular}{|c|c|c|c|c|c|}
\hline & Algarve (Portugal) & 0.0120792 & - & 1 & 0 \\
\hline & Madeira (Portugal) & 0.0182472 & - & 1 & 0 \\
\hline & Naples (Italy) & 0.003084 & - & 1 & 0 \\
\hline & Crete (Greece) & 0.0102801 & - & 1 & 0 \\
\hline & Kos (Greece) & 0.001799 & - & 1 & 0 \\
\hline & Mikonos (Greece) & 0.0095091 & - & 1 & 0 \\
\hline & Naxos (Greece) & 0.001285 & - & 1 & 0 \\
\hline & Paros (Greece) & 0.002056 & - & 1 & 0 \\
\hline & Rhodes (Greece) & 0.0038551 & - & 1 & 0 \\
\hline & Santorini (Greece) & 0.0095091 & - & 1 & 0 \\
\hline & Skiathos (Greece) & 0.000771 & - & 1 & 0 \\
\hline Tour operator & Rhodasol & 0.0881521 & - & 1 & 0 \\
\hline & Travelplan & 0.2109997 & - & 1 & 0 \\
\hline & Mundicolor & 0.1362118 & - & 1 & 0 \\
\hline & Vivatours & 0.0701619 & - & 1 & 0 \\
\hline & Viajes El Corte Inglés & 0.1719352 & - & 1 & 0 \\
\hline & Iberojet & 0.2038036 & - & 1 & 0 \\
\hline & Marsol & 0.1187355 & - & 1 & 0 \\
\hline
\end{tabular}

Mean Standard Maximum Minimum

deviation

with six different specifications. Thus the following regression functions are estimated when seasonality is captured with the coefficient of variation:

$$
\begin{aligned}
& C V p_{b}=f\left(\mathbf{c}_{b}, l_{b}, t_{b} ; \boldsymbol{\beta}\right)+u \\
& \log C V p_{b}=f\left(\mathbf{c}_{b}, l_{b}, t_{b} ; \boldsymbol{\beta}\right)+u,
\end{aligned}
$$

when the seasonality ratio is used the specifications are the following:

$$
\begin{aligned}
& S R p_{b}=f\left(\mathbf{c}_{b}, l_{b}, t_{b} ; \boldsymbol{\beta}\right)+u \\
& \log S R p_{b}=f\left(\mathbf{c}_{b}, l_{b}, t_{b} ; \boldsymbol{\beta}\right)+u,
\end{aligned}
$$

and when the maximum price in the season divided by the minimum price is the measure of seasonality, we have:

$$
\begin{aligned}
& S p_{b}=f\left(\mathbf{c}_{b}, l_{b}, t_{b} ; \boldsymbol{\beta}\right)+u \\
& \log S p_{b}=f\left(\mathbf{c}_{b}, l_{b}, t_{b} ; \boldsymbol{\beta}\right)+u,
\end{aligned}
$$

where 'log' is the natural logarithm, $f(\cdot)$ is a linear function of the parameters, $u$ is a random error term and the rest of the elements have already been defined in the previous section. 


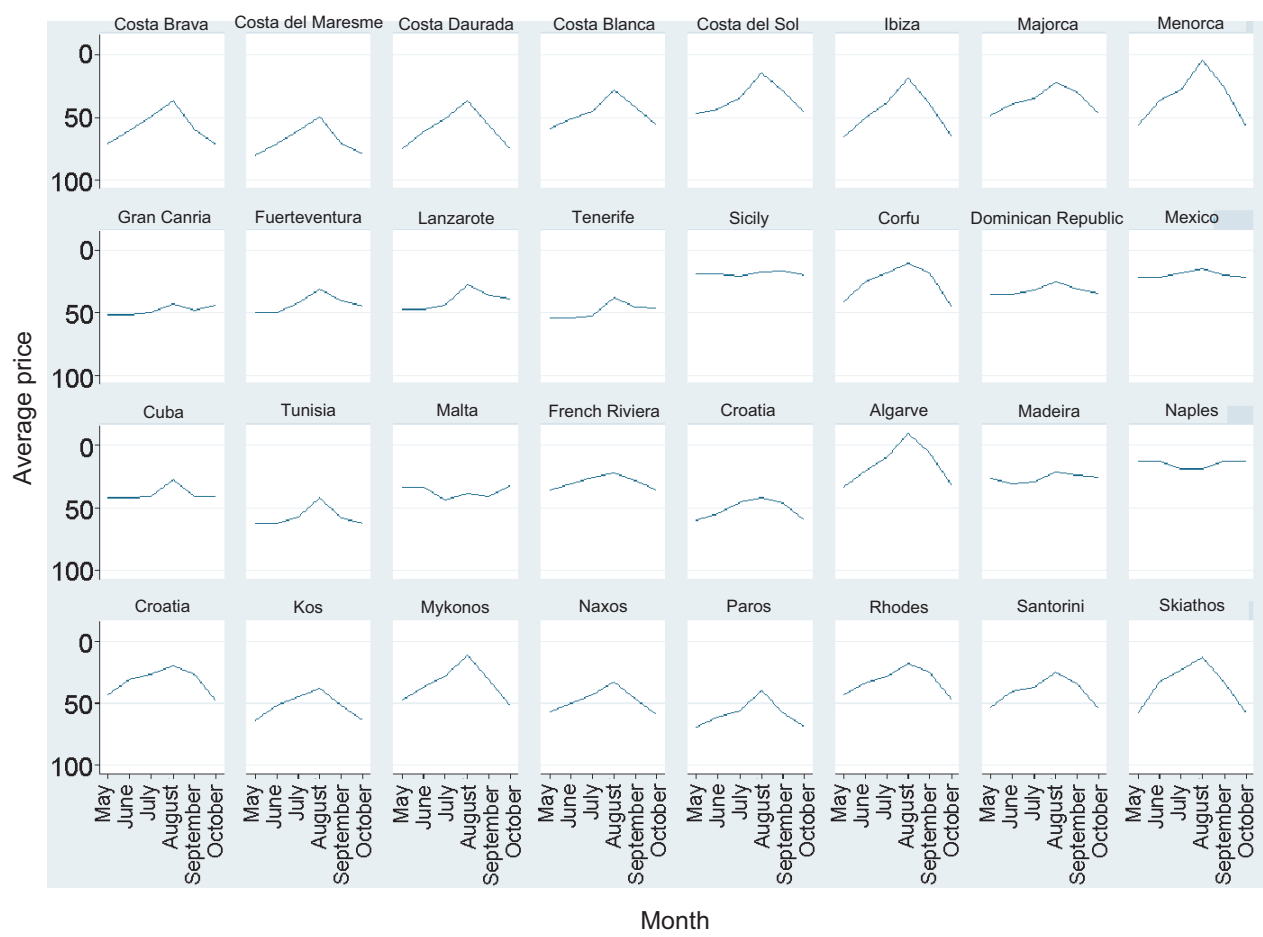

Figure 1. Monthly average prices for the destinations in the sample.

\section{Results}

Table 3 shows the results of estimating the specifications (6)-(11) using White's (1980) heteroskedasticity robust estimator of the variance-covariance matrix. The estimates are generally robust to the changes in the dependent variable. The adjusted- $R^{2}$ for the different regressions (which range from 0.5778 and 0.7064 ) indicate that the fit is very good for all the alternative specifications. Many variables are individually significant at very strong levels, and the hypothesis that the slope coefficients in the specifications are jointly zero can be rejected (the $p$ value for the $F$ test is smaller than 0.001 for all the specifications). Multicollinearity is not a problem since the mean VIF (variance inflation factor) is 1.81 and the largest VIF (6.02) is lower than 10.

The estimates of the coefficients linked to hotel services and characteristics in Table 3 provide interesting information for managers. On the one hand, the availability of room services and outdoor and indoor sports facilities are 
Table 3. Results for the different specifications of the model.

Dependent variable

\begin{tabular}{|c|c|c|c|c|c|}
\hline $\begin{array}{c}\text { Coefficient } \\
\text { of variation } \\
\text { of prices } \\
\text { in levels }\end{array}$ & $\begin{array}{l}\text { Coefficient } \\
\text { of variation } \\
\text { of prices } \\
\text { in logs }\end{array}$ & $\begin{array}{l}\text { Seasonality } \\
\text { ratio in } \\
\text { levels }\end{array}$ & $\begin{array}{l}\text { Seasonality } \\
\text { ratio in } \\
\log s\end{array}$ & $\begin{array}{c}\text { Maximum } \\
\text { price over } \\
\text { minimum } \\
\text { price in } \\
\text { levels }\end{array}$ & $\begin{array}{l}\text { Maximum } \\
\text { price over } \\
\text { minimum } \\
\text { price in } \\
\text { logs }\end{array}$ \\
\hline
\end{tabular}

Services available at the hotel:

\begin{tabular}{|c|c|c|c|c|c|c|}
\hline $\begin{array}{l}\text { Hotel located in front } \\
\text { of beach }\end{array}$ & $\begin{array}{l}-0.0008 \\
(0.0029)\end{array}$ & $\begin{array}{c}0.0129 \\
(0.0172)\end{array}$ & $\begin{array}{l}-0.0013 \\
(0.0046)\end{array}$ & $\begin{array}{l}-0.0005 \\
(0.0034)\end{array}$ & $\begin{array}{c}0.0093 \\
(0.0151)\end{array}$ & $\begin{array}{c}0.0036 \\
(0.0070)\end{array}$ \\
\hline Room services available & $\begin{array}{c}-0.0151^{* * *} \\
(0.0045)\end{array}$ & $\begin{array}{c}-0.0797^{* * *} \\
(0.0238)\end{array}$ & $\begin{array}{c}-0.0276^{* * *} \\
(0.0080)\end{array}$ & $\begin{array}{c}-0.0202^{* * *} \\
(0.0056)\end{array}$ & $\begin{array}{l}-0.0489^{*} \\
(0.0242)\end{array}$ & $\begin{array}{c}-0.0311^{* *} \\
(0.0113)\end{array}$ \\
\hline $\begin{array}{l}\text { Garden or balcony } \\
\text { available }\end{array}$ & $\begin{array}{c}0.0078 \\
(0.0072)\end{array}$ & $\begin{array}{l}0.1077^{*} \\
(0.0456)\end{array}$ & & $\begin{array}{c}0.0126 \\
(0.0077)\end{array}$ & & $\begin{array}{l}0.0311^{*} \\
(0.0156)\end{array}$ \\
\hline $\begin{array}{l}\text { Swimming pool } \\
\text { available }\end{array}$ & $\begin{array}{l}0.0365^{* * *} \\
(0.0087)\end{array}$ & $\begin{array}{l}0.1985^{* * *} \\
(0.0571)\end{array}$ & $\begin{array}{l}0.0525^{* * * *} \\
(0.0128)\end{array}$ & $\begin{array}{l}0.0392^{* * *} \\
(0.0092)\end{array}$ & $\begin{array}{l}0.1954^{* * *} \\
(0.0358)\end{array}$ & $\begin{array}{l}0.1013^{* * *} \\
(0.0180)\end{array}$ \\
\hline $\begin{array}{l}\text { Outdoor sports facilities } \\
\text { available }\end{array}$ & $\begin{array}{l}-0.0074^{*} \\
(0.0029)\end{array}$ & $\begin{array}{l}-0.0065 \\
(0.0171)\end{array}$ & & & $\begin{array}{l}-0.0336^{*} \\
(0.0151)\end{array}$ & $\begin{array}{l}-0.0116 \\
(0.0071)\end{array}$ \\
\hline Car park available & $\begin{array}{l}-0.0042 \\
(0.0034)\end{array}$ & $\begin{array}{l}-0.0451^{*} \\
(0.0209)\end{array}$ & $\begin{array}{l}-0.0070 \\
(0.0050)\end{array}$ & $\begin{array}{l}-0.0066 \\
(0.0037)\end{array}$ & $\begin{array}{c}0.0087 \\
(0.0180)\end{array}$ & $\begin{array}{l}-0.0034 \\
(0.0078)\end{array}$ \\
\hline $\begin{array}{l}\text { Animation activities } \\
\text { available }\end{array}$ & $\begin{array}{l}0.0420^{* * * *} \\
(0.0029)\end{array}$ & $\begin{array}{l}0.2346^{* * *} \\
(0.0171)\end{array}$ & $\begin{array}{l}0.0720^{* * * *} \\
(0.0047)\end{array}$ & $\begin{array}{l}0.0532^{* * *} \\
(0.0034)\end{array}$ & $\begin{array}{l}0.1859^{* * *} \\
(0.0156)\end{array}$ & $\begin{array}{l}0.1027^{* * *} \\
(0.0073)\end{array}$ \\
\hline $\begin{array}{l}\text { Indoor sports facilities } \\
\text { available }\end{array}$ & $\begin{array}{l}-0.0062^{*} \\
(0.0031)\end{array}$ & $\begin{array}{l}-0.0057 \\
(0.0192)\end{array}$ & $\begin{array}{l}-0.0145^{* *} \\
(0.0049)\end{array}$ & $\begin{array}{l}-0.0091^{*} \\
(0.0036)\end{array}$ & $\begin{array}{l}-0.0579^{* * *} \\
(0.0149)\end{array}$ & $\begin{array}{l}-0.0223^{* *} \\
(0.0074)\end{array}$ \\
\hline $\begin{array}{l}\text { Hotel has been } \\
\text { refurbished }\end{array}$ & $\begin{array}{c}0.0050 \\
(0.0026)\end{array}$ & $\begin{array}{l}0.0356^{*} \\
(0.0162)\end{array}$ & $\begin{array}{l}0.0101^{*} \\
(0.0043)\end{array}$ & $\begin{array}{l}0.0081^{*} \\
(0.0032)\end{array}$ & $\begin{array}{c}0.0162 \\
(0.0133)\end{array}$ & $\begin{array}{c}0.0106 \\
(0.0064)\end{array}$ \\
\hline \multicolumn{7}{|l|}{ Hotel star rating: } \\
\hline 1-star hotel & $\begin{array}{l}0.0272^{*} \\
(0.0124)\end{array}$ & $\begin{array}{l}0.2325^{* * *} \\
(0.0576)\end{array}$ & $\begin{array}{c}0.0262 \\
(0.0236)\end{array}$ & $\begin{array}{c}0.0255 \\
(0.0162)\end{array}$ & $\begin{array}{c}0.0547 \\
(0.0704)\end{array}$ & $\begin{array}{c}0.0579 \\
(0.0333)\end{array}$ \\
\hline 2-star hotel & $\begin{array}{l}-0.0011 \\
(0.0074)\end{array}$ & $\begin{array}{c}0.0295 \\
(0.0360)\end{array}$ & $\begin{array}{l}-0.0048 \\
(0.0125)\end{array}$ & $\begin{array}{l}-0.0012 \\
(0.0087)\end{array}$ & $\begin{array}{l}-0.0642 \\
(0.0374)\end{array}$ & $\begin{array}{l}-0.0195 \\
(0.0179)\end{array}$ \\
\hline tel & $\begin{array}{c}-0.0503^{* * *} \\
(0.0035)\end{array}$ & $\begin{array}{c}-0.2388^{* * *} \\
(0.0226)\end{array}$ & $\begin{array}{c}-0.0693^{* * *} \\
(0.0053)\end{array}$ & $\begin{array}{c}-0.0504^{* * *} \\
(0.0039)\end{array}$ & $\begin{array}{c}-0.2713^{* * *} \\
(0.0161)\end{array}$ & $\begin{array}{c}-0.1371^{* * *} \\
(0.0082)\end{array}$ \\
\hline -star hotel & $\begin{array}{c}-0.0861^{* * *} \\
(0.0054)\end{array}$ & $\begin{array}{c}-0.5085^{* * *} \\
(0.0401)\end{array}$ & $\begin{array}{c}-0.1346^{* * *} \\
(0.0078)\end{array}$ & $\begin{array}{c}-0.1035^{* * *} \\
(0.0061)\end{array}$ & $\begin{array}{c}-0.3881^{* * *} \\
(0.0266)\end{array}$ & $\begin{array}{c}-0.2288^{* * *} \\
(0.0126)\end{array}$ \\
\hline
\end{tabular}

Accommodation type (reference - half board):

$\begin{array}{lcccccc}\text { Lunch included } & -0.0393^{*} & -0.2835 & -0.0190 & -0.0196 & -0.0204 & -0.0479 \\ & (0.0168) & (0.1968) & (0.0236) & (0.0191) & (0.0688) & (0.0363) \\ \text { Breakfast included } & -0.0265 & -0.1023 & -0.0015 & -0.0052 & 0.0454 & -0.0178 \\ & (0.0198) & (0.1233) & (0.0174) & (0.0137) & (0.0801) & (0.0328) \\ \text { Full board } & -0.1148^{* * *} & -0.8768^{* * *} & -0.0924^{* *} & -0.0724^{* * *} & -0.1736^{*} & -0.1220^{* *} \\ & (0.0244) & (0.1934) & (0.0287) & (0.0220) & (0.0801) & (0.0462) \\ \text { All inclusive } & -0.0389^{* *} & -0.3058^{* * *} & -0.0297 & -0.0213 & -0.0664 & -0.0535^{*} \\ & (0.0129) & (0.0896) & (0.0167) & (0.0130) & (0.0563) & (0.0256) \\ \text { Hotel type: } & & & & & & \\ \text { Apartment hotel } & -0.0086 & -0.0437 & -0.0297^{* * *} & -0.0184^{* * *} & -0.0603^{*} & -0.0281^{*} \\ & (0.0049) & (0.0279) & (0.0074) & (0.0054) & (0.0240) & (0.0119) \\ \text { Region (reference -Costa Brava, Spain): } & & & & & \\ \text { Costa del Maresme } & 0.0766^{* * *} & 0.2586^{* * *} & 0.1198^{* * *} & 0.0815^{* * *} & 0.4379^{* * *} & 0.2018^{* * *} \\ \text { (Spain) } & (0.0049) & (0.0202) & (0.0090) & (0.0061) & (0.0283) & (0.0124) \\ \text { Costa Daurada (Spain) } & 0.0798^{* * * *} & 0.2488^{* * *} & 0.0899^{* * *} & 0.0612^{* * *} & 0.6191^{* * *} & 0.2575^{* * *} \\ & (0.0058) & (0.0285) & (0.0098) & (0.0067) & (0.0378) & (0.0155)\end{array}$


Table 3 continued.

Dependent variable

$\begin{array}{cccccc}\begin{array}{c}\text { Coefficient } \\ \text { of variation }\end{array} & \begin{array}{c}\text { Coefficient } \\ \text { of variation }\end{array} & \begin{array}{c}\text { Seasonality } \\ \text { ratio in }\end{array} & \begin{array}{c}\text { Seasonality } \\ \text { ratio in }\end{array} & \begin{array}{c}\text { Maximum } \\ \text { price over }\end{array} & \begin{array}{c}\text { Maximum } \\ \text { price over }\end{array} \\ \text { of prices } & \text { of prices } & \text { levels } & \operatorname{logs} & \begin{array}{c}\text { minimum } \\ \text { minimum }\end{array} \\ \text { in levels } & \text { in logs } & & & \text { price in } & \text { price in } \\ & & & & \text { levels } & \text { logs }\end{array}$

\begin{tabular}{|c|c|c|c|c|c|c|}
\hline Costa Blanca (Spain) & $\begin{array}{c}-0.0583^{* * *} \\
(0.0048)\end{array}$ & $\begin{array}{c}-0.2241^{* * *} \\
(0.0289)\end{array}$ & $\begin{array}{c}-0.0850^{* * *} \\
(0.0080)\end{array}$ & $\begin{array}{c}-0.0566^{* * *} \\
(0.0058)\end{array}$ & $\begin{array}{c}-0.2135^{* * *} \\
(0.0240)\end{array}$ & $\begin{array}{c}-0.0929^{* * *} \\
(0.0124)\end{array}$ \\
\hline \multirow[t]{2}{*}{ Costa del Sol (Spain) } & 0.0004 & 0.0201 & -0.0053 & -0.0024 & -0.0289 & -0.0119 \\
\hline & $(0.0054)$ & $(0.0300)$ & $(0.0090)$ & $(0.0065)$ & $(0.0297)$ & $(0.0142)$ \\
\hline \multirow[t]{2}{*}{ Ibiza (Spain) } & $0.0475^{* * *}$ & $0.1425^{* * *}$ & $0.0510^{* * *}$ & $0.0339^{* * *}$ & $0.3178^{* * *}$ & $0.1396^{* * * *}$ \\
\hline & $(0.0076)$ & $(0.0304)$ & $(0.0140)$ & (0.0095) & $(0.0440)$ & $(0.0187)$ \\
\hline \multirow[t]{2}{*}{ Majorca (Spain) } & $-0.0838^{* * *}$ & $-0.3856^{* * *}$ & $-0.1645^{* * *}$ & $-0.1202^{* * *}$ & $-0.3893^{* * *}$ & $-0.2013^{* * *}$ \\
\hline & $(0.0057)$ & $(0.0332)$ & $(0.0091)$ & $(0.0067)$ & $(0.0268)$ & $(0.0141)$ \\
\hline \multirow[t]{2}{*}{ Menorca (Spain) } & $0.0188^{*}$ & $0.0824^{*}$ & 0.0083 & 0.0065 & $0.2101^{* *}$ & $0.0905^{* * *}$ \\
\hline & $(0.0092)$ & $(0.0333)$ & $(0.0149)$ & (0.0099) & $(0.0694)$ & $(0.0268)$ \\
\hline \multirow[t]{2}{*}{ Gran Canaria (Spain) } & $-0.1614^{* * *}$ & $-0.8547^{* * *}$ & $-0.2499^{* * *}$ & $-0.1877^{* * *}$ & $-0.7113^{* * *}$ & $-0.4013^{* * *}$ \\
\hline & $(0.0063)$ & $(0.0443)$ & (0.0109) & $(0.0083)$ & $(0.0268)$ & $(0.0149)$ \\
\hline \multirow[t]{2}{*}{ Fuerteventura (Spain) } & $-0.1322^{* * *}$ & $-0.7106^{* * *}$ & $-0.2085^{* * *}$ & $-0.1564^{* * * *}$ & $-0.6132^{* * *}$ & $-0.3390^{* * *}$ \\
\hline & $(0.0101)$ & $(0.0638)$ & $(0.0178)$ & $(0.0129)$ & & $(0.0231)$ \\
\hline \multirow[t]{2}{*}{ Lanzarote (Spain) } & $-0.1332^{* * *}$ & $-0.6316^{* * *}$ & $-0.2031^{\text {*** }}$ & $-0.1488^{* * *}$ & $-0.6226^{* * *}$ & $-0.3336^{* * *}$ \\
\hline & $(0.0066)$ & $(0.0395)$ & $(0.0111)$ & $(0.0084)$ & & 69) \\
\hline \multirow[t]{2}{*}{ Tenerife (Spain) } & $-0.1130^{* * *}$ & $-0.5533^{* * *}$ & $-0.1617^{* * *}$ & $-0.1173^{* * *}$ & $-0.5392^{* * *}$ & $-0.2881^{\text {**** }}$ \\
\hline & $(0.0056)$ & $(0.0356)$ & $(0.0097)$ & $(0.0073)$ & $(0.0256)$ & $(0.0136)$ \\
\hline \multirow[t]{2}{*}{ Sicily (Italy) } & 0.0185 & -0.2235 & $-0.2528^{* * *}$ & $-0.1896^{* * *}$ & $-0.7833^{* * *}$ & $-0.4508^{\text {**** }}$ \\
\hline & $(0.0265)$ & $(0.1823)$ & $(0.0187)$ & $(0.0149)$ & $(0.0696)$ & $(0.0369)$ \\
\hline \multirow[t]{2}{*}{ Corfu (Greece) } & $-0.0375^{*}$ & -0.0808 & $-0.1608^{* * *}$ & $-0.1123^{* * *}$ & $-0.2350^{* * *}$ & $-0.0809^{*}$ \\
\hline & $(0.0179)$ & $(0.0954)$ & $(0.0275)$ & $(0.0220)$ & $(0.0682)$ & $(0.0388)$ \\
\hline \multirow[t]{2}{*}{ Dominican Republic } & $-0.1487^{* * *}$ & $-0.8213^{* * *}$ & $-0.2545^{* * *}$ & $-0.1977^{* * *}$ & $-0.7037^{* * *}$ & $-0.4092^{* * *}$ \\
\hline & $(0.0147)$ & $(0.1084)$ & $(0.0192)$ & $(0.0152)$ & $(0.0603)$ & $(0.0292)$ \\
\hline \multirow[t]{2}{*}{ Mexico } & $-0.1545^{* * *}$ & $-0.9203^{* * *}$ & $-0.2719^{* * *}$ & $-0.2142^{* * *}$ & $-0.7248^{* * *}$ & $-0.4276^{* * *}$ \\
\hline & $(0.0149)$ & $(0.1104)$ & $(0.0186)$ & $(0.0145)$ & $(0.0620)$ & $(0.0297)$ \\
\hline \multirow[t]{2}{*}{ Cuba } & $-0.1239^{* * *}$ & $-0.6513^{* * *}$ & $-0.1803^{* * *}$ & $-0.1314^{* * *}$ & $-0.6461^{* * *}$ & $-0.3499^{* * *}$ \\
\hline & $(0.0134)$ & $(0.0857)$ & $(0.0164)$ & $(0.0127)$ & $(0.0540)$ & $(0.0255)$ \\
\hline \multirow[t]{2}{*}{ Tunisia } & $-0.0230^{*}$ & 0.1048 & -0.0072 & 0.0020 & $-0.3229^{* * *}$ & $-0.1386^{* * *}$ \\
\hline & $(0.0116)$ & $(0.0709)$ & $(0.0186)$ & $(0.0137)$ & & $(0.0248)$ \\
\hline \multirow[t]{2}{*}{ Malta } & $-0.1298^{* * *}$ & $-1.1126^{* * *}$ & $-0.2650^{* * *}$ & $-0.2049^{* * *}$ & -0.4657 & $-0.3563^{* * *}$ \\
\hline & $(0.0343)$ & $(0.1852)$ & & $(0.0251)$ & & \\
\hline \multirow[t]{2}{*}{ French Riviera (France) } & $-0.1377^{* * *}$ & $-1.0002^{* * *}$ & $-0.2982^{* * *}$ & $-0.2239^{* * *}$ & $-0.7623^{* * *}$ & $-0.4026^{* * *}$ \\
\hline & $(0.0238)$ & & (0.0269) & $(0.0219)$ & & $(0.0477)$ \\
\hline \multirow[t]{2}{*}{ Croatia } & $-0.0853^{* * *}$ & $-0.2938^{* *}$ & $-0.2185^{* * *}$ & $-0.1567^{* * *}$ & $-0.5475^{* * *}$ & $-0.2672^{* * *}$ \\
\hline & $(0.0187)$ & & $(0.0249)$ & $(0.0196)$ & & $(0.0439)$ \\
\hline \multirow[t]{2}{*}{ Algarve (Portugal) } & $-0.0608^{* * *}$ & $-0.1684^{*}$ & $-0.1482^{* * *}$ & $-0.1020^{* * * *}$ & $-0.3633^{* * *}$ & $-0.1463^{\text {*** }}$ \\
\hline & $(0.0136)$ & $(0.0775)$ & $(0.0175)$ & $(0.0133)$ & & $(0.0282)$ \\
\hline \multirow[t]{2}{*}{ Madeira (Portugal) } & $-0.1786^{* * *}$ & $-1.4877^{* * *}$ & $-0.3123^{* * *}$ & $-0.2453^{* * *}$ & $-0.7841^{* * *}$ & $-0.4654^{* * * *}$ \\
\hline & $(0.0131)$ & & & $(0.0118)$ & & $(0.0256)$ \\
\hline \multirow[t]{2}{*}{ Naples (Italy) } & $-0.1778^{* * *}$ & $-1.2280^{* * *}$ & $-0.3377^{* * *}$ & $-0.2659^{* * *}$ & $-0.7866^{* * *}$ & $-0.4454^{* * *}$ \\
\hline & $(0.0273)$ & $(0.2509)$ & & $(0.0231)$ & & $(0.0533)$ \\
\hline \multirow[t]{2}{*}{ Crete (Greece) } & $-0.0785^{* * *}$ & $-0.3591^{* * *}$ & $-0.2285^{* * *}$ & $-0.1672^{* * *}$ & $-0.4398^{* * *}$ & $-0.2075^{* * *}$ \\
\hline & $(0.0146)$ & $(0.1028)$ & & $(0.0154)$ & & $(0.0335)$ \\
\hline \multirow[t]{2}{*}{ Kos (Greece) } & $-0.0403^{*}$ & -0.0107 & $-0.1755^{* * *}$ & $-0.1232^{* * *}$ & $-0.3599^{* * *}$ & $-0.1545^{* *}$ \\
\hline & $(0.0205)$ & $(0.1229)$ & $(0.0354)$ & $(0.0280)$ & $(0.0986)$ & $(0.0550)$ \\
\hline
\end{tabular}


Table 3 continued.

\section{Dependent variable}

\begin{tabular}{|c|c|c|c|c|c|}
\hline $\begin{array}{c}\text { Coefficient } \\
\text { of variation } \\
\text { of prices } \\
\text { in levels }\end{array}$ & $\begin{array}{l}\text { Coefficient } \\
\text { of variation } \\
\text { of prices } \\
\text { in logs }\end{array}$ & $\begin{array}{l}\text { Seasonality } \\
\text { ratio in } \\
\text { levels }\end{array}$ & $\begin{array}{l}\text { Seasonality } \\
\text { ratio in } \\
\operatorname{logs}\end{array}$ & $\begin{array}{l}\text { Maximum } \\
\text { price over } \\
\text { minimum } \\
\text { price in } \\
\text { levels }\end{array}$ & $\begin{array}{l}\text { Maximum } \\
\text { price over } \\
\text { minimum } \\
\text { price in } \\
\text { logs }\end{array}$ \\
\hline
\end{tabular}

\begin{tabular}{|c|c|c|c|c|c|c|}
\hline Mykonos (Greece) & $\begin{array}{c}0.0353 \\
(0.0225)\end{array}$ & $\begin{array}{c}0.2547^{*} \\
(0.1184)\end{array}$ & $\begin{array}{l}-0.0054 \\
(0.0274)\end{array}$ & $\begin{array}{c}0.0070 \\
(0.0201)\end{array}$ & $\begin{array}{c}0.0474 \\
(0.1008)\end{array}$ & $\begin{array}{c}0.0806 \\
(0.0460)\end{array}$ \\
\hline Naxos (Greece) & $\begin{array}{c}0.0053 \\
(0.0249)\end{array}$ & $\begin{array}{c}0.1332 \\
(0.1498)\end{array}$ & $\begin{array}{l}-0.0010 \\
(0.0499)\end{array}$ & $\begin{array}{c}0.0115 \\
(0.0392)\end{array}$ & $\begin{array}{l}-0.1166 \\
(0.0948)\end{array}$ & $\begin{array}{c}0.0111 \\
(0.0561)\end{array}$ \\
\hline Paros (Greece) & $\begin{array}{c}0.0283 \\
(0.0326)\end{array}$ & $\begin{array}{c}0.1919 \\
(0.1408)\end{array}$ & $\begin{array}{c}0.0425 \\
(0.0439)\end{array}$ & $\begin{array}{c}0.0436 \\
(0.0303)\end{array}$ & $\begin{array}{l}-0.0795 \\
(0.1265)\end{array}$ & $\begin{array}{c}0.0243 \\
(0.0644)\end{array}$ \\
\hline Rhodes (Greece) & $\begin{array}{c}-0.0453^{*} \\
(0.0182)\end{array}$ & $\begin{array}{l}-0.1386 \\
(0.1143)\end{array}$ & $\begin{array}{c}-0.1481^{* * *} \\
(0.0260)\end{array}$ & $\begin{array}{c}-0.1033^{* * *} \\
(0.0208)\end{array}$ & $\begin{array}{c}-0.2607^{* *} \\
(0.0798)\end{array}$ & $\begin{array}{c}-0.0980^{*} \\
(0.0440)\end{array}$ \\
\hline Santorini (Greece) & $\begin{array}{l}-0.0369 \\
(0.0201)\end{array}$ & $\begin{array}{l}-0.0671 \\
(0.1197)\end{array}$ & $\begin{array}{c}-0.1260^{* * *} \\
(0.0233)\end{array}$ & $\begin{array}{c}-0.0822^{* * *} \\
(0.0182)\end{array}$ & $\begin{array}{c}-0.3100^{* * *} \\
(0.0789)\end{array}$ & $\begin{array}{r}-0.1038^{* *} \\
(0.0398)\end{array}$ \\
\hline Skiathos (Greece) & $\begin{array}{c}0.0941^{* * *} \\
(0.0274)\end{array}$ & $\begin{array}{l}0.6132^{* * *} \\
(0.1759)\end{array}$ & $\begin{array}{c}0.0378 \\
(0.0318)\end{array}$ & $\begin{array}{c}0.0491 \\
(0.0251)\end{array}$ & $\begin{array}{c}0.1687 \\
(0.1179)\end{array}$ & $\begin{array}{l}0.2004^{* *} \\
(0.0631)\end{array}$ \\
\hline Tour operator (reference - & (arsol): & & & & & \\
\hline Rhodasol & $\begin{array}{l}-0.0022 \\
(0.0057)\end{array}$ & $\begin{array}{c}0.0062 \\
(0.0298)\end{array}$ & $\begin{array}{c}0.0006 \\
(0.0098)\end{array}$ & $\begin{array}{c}0.0008 \\
(0.0069)\end{array}$ & $\begin{array}{l}-0.0110 \\
(0.0317)\end{array}$ & $\begin{array}{l}-0.0041 \\
(0.0147)\end{array}$ \\
\hline Travelplan & $\begin{array}{l}-0.0041 \\
(0.0052)\end{array}$ & $\begin{array}{l}-0.0056 \\
(0.0273)\end{array}$ & $\begin{array}{l}-0.0109 \\
(0.0090)\end{array}$ & $\begin{array}{l}-0.0085 \\
(0.0063)\end{array}$ & $\begin{array}{l}-0.0113 \\
(0.0284)\end{array}$ & $\begin{array}{l}-0.0072 \\
(0.0134)\end{array}$ \\
\hline Mundicolor & $\begin{array}{c}-0.0143^{*} \\
(0.0060)\end{array}$ & $\begin{array}{l}-0.0747^{*} \\
(0.0368)\end{array}$ & $\begin{array}{c}-0.0345^{* * *} \\
(0.0099)\end{array}$ & $\begin{array}{c}-0.0285^{* * *} \\
(0.0072)\end{array}$ & $\begin{array}{l}-0.0407 \\
(0.0305)\end{array}$ & $\begin{array}{c}-0.0319^{*} \\
(0.0149)\end{array}$ \\
\hline Vivatours & $\begin{array}{c}-0.0174^{*} \\
(0.0069)\end{array}$ & $\begin{array}{l}-0.0472 \\
(0.0464)\end{array}$ & $\begin{array}{c}-0.0316^{* *} \\
(0.0107)\end{array}$ & $\begin{array}{c}-0.0250^{* *} \\
(0.0080)\end{array}$ & $\begin{array}{l}-0.0382 \\
(0.0306)\end{array}$ & $\begin{array}{l}-0.0304 \\
(0.0157)\end{array}$ \\
\hline Viajes El Corte Inglés & $\begin{array}{l}0.0221^{* * *} \\
(0.0056)\end{array}$ & $\begin{array}{c}0.1252^{* * *} \\
(0.0314)\end{array}$ & $\begin{array}{c}0.0141 \\
(0.0091)\end{array}$ & $\begin{array}{c}0.0115 \\
(0.0065)\end{array}$ & $\begin{array}{c}0.0425 \\
(0.0300)\end{array}$ & $\begin{array}{c}0.0199 \\
(0.0139)\end{array}$ \\
\hline Iberojet & $\begin{array}{l}0.0142^{* *} \\
(0.0052)\end{array}$ & $\begin{array}{l}0.0686^{*} \\
(0.0283)\end{array}$ & $\begin{array}{l}0.0346^{* * *} \\
(0.0088)\end{array}$ & $\begin{array}{c}0.0265^{* * *} \\
(0.0062)\end{array}$ & $\begin{array}{c}0.1060^{* * *} \\
(0.0290)\end{array}$ & $\begin{array}{c}0.0581^{* * *} \\
(0.0132)\end{array}$ \\
\hline Hotel chain type: & & & & & & \\
\hline Chain & $\begin{array}{c}0.0044 \\
(0.0028)\end{array}$ & $\begin{array}{c}0.0132 \\
(0.0191)\end{array}$ & $\begin{array}{c}0.0004 \\
(0.0044)\end{array}$ & $\begin{array}{c}0.0020 \\
(0.0033)\end{array}$ & $\begin{array}{l}0.0449^{* *} \\
(0.0154)\end{array}$ & $\begin{array}{c}0.0235^{* * * *} \\
(0.0071)\end{array}$ \\
\hline Constant & $\begin{array}{l}0.2676^{* * *} \\
(0.0103)\end{array}$ & $\begin{array}{c}-1.5555^{* * *} \\
(0.0758)\end{array}$ & $\begin{array}{l}1.4282^{* * * *} \\
(0.0157)\end{array}$ & $\begin{array}{c}0.3417^{\text {**** }} \\
(0.0114)\end{array}$ & $\begin{array}{l}1.9545^{* * *} \\
(0.0451)\end{array}$ & $\begin{array}{c}0.6185^{\text {*** }} \\
(0.0229)\end{array}$ \\
\hline Regression statistics: & & & & & & \\
\hline$N$ & 3,891 & 3,765 & 3,891 & 3,891 & 3,855 & 3,855 \\
\hline Adjusted $R$-squared & 0.6527 & 0.5778 & 0.6517 & 0.6622 & 0.6535 & 0.7064 \\
\hline$F$ & 224.1668 & 120.7898 & 182.8716 & 188.3202 & 196.6764 & 248.1968 \\
\hline$P$ value & 0.0000 & 0.0000 & 0.0000 & 0.0000 & 0.0000 & 0.0000 \\
\hline Mean VIF & \multicolumn{6}{|c|}{1.81} \\
\hline Max VIF & \multicolumn{6}{|c|}{6.02} \\
\hline
\end{tabular}

Note: Robust standard errors are in parentheses. ${ }^{*} p<0.05 ;{ }^{* *} p<0.01 ;{ }^{* * * *} p<0.001$. 
negatively related to seasonality. On the other hand, the availability of a swimming pool and animation activities, as well as recent refurbishments, are positively associated with seasonality. Finally, location in front of the beach, the availability of a garden or a balcony in the room and the availability of a car park are not statistically related to seasonality in prices. As to the hotel star rating, higher star ratings are associated with lower seasonality in prices. The estimates for the different types of accommodation do not show a clear pattern, since all types of accommodation are negatively associated with seasonality when compared to half board (although the results for 'lunch included' and 'breakfast included' are essentially not significant statistically). Apartment hotels are negatively associated with seasonality when compared to hotels. Finally, hotels belonging to a chain show higher levels of seasonality in prices than independent hotels. The implications of these results are discussed in detail in the next section.

Before proceeding, it is worthwhile to compare seasonality in prices across the destinations considered. Table 4 shows six rankings of destinations according to seasonality computed by using the estimates in Table 3 which are associated with the destination variables in (6)-(11). The seasonality index for destination $i$ can be computed as $100 \cdot e^{\hat{\beta}_{\text {destination }}}$, where $\hat{\beta}_{\text {destination } i}$ is the estimate of the parameter associated with destination $i$ in Table 3 . The reference destination (Costa Brava) takes the value 100. The rankings obtained from the estimates of equations (6)-(11) are highly similar to each other: the pairwise Pearson correlation coefficients between the different rankings range from 0.921 to 0.997 . As a rule, destinations in the Mediterranean show a higher degree of seasonality in prices than those in the Caribbean, the Canary Islands and Madeira. This shows that proximity to the equator, besides the well-known fact that it diminishes seasonality in the number of tourists (see Baum and Lundtorp, 2001, for instance), also diminishes seasonality in prices. Nevertheless, there are notable exceptions to this rule, such as Malta, the French Riviera and Naples, suggesting that other factors besides the climate and hotel services and star ratings also play a role in explaining seasonality. This is discussed in more detail in the next section.

\section{Discussion}

The estimates in Table 3 show the relationship between different variables and seasonality. As is well-known, correlation does not mean causation. Nevertheless, the estimates provide some hints for managers of tourism firms and destinations about how it might be possible to smooth the seasonality observed in hotel prices.

\section{Hotel characteristics and seasonality}

The finding that, other things being equal, higher hotel star ratings are associated with lower seasonality is not trivial, since a priori the causeconsequence relationship may go both ways. That is, it is possible that hotels with higher star ratings decide to locate in destinations (close to the equator, for instance) where seasonality is expected to be lower. Indeed, the paper's 
finding is useful for policy makers and managers since, when considered together with the negative association between seasonality and the availability of room service, it implies that providing products of higher quality and/or with more services embedded paves the way for diminishing seasonality in prices. This conclusion is similar to that of Capó Parrilla et al (2007) when analysing seasonality in the number of visitors. Nevertheless, our results provide an interesting new insight: the increase in the number of visitors which accompanies the increase in the quantity/quality of services (as found by Capó Parrilla et al, 2007) need not come at the cost of lower prices, since (as found here) there is a negative correlation between both hotels' star ratings and the services available and seasonality in prices. Therefore, increasing the quality of hotel accommodation appears to be a promising way to reduce seasonality in prices. As long as the cost of improving quality is not too high, the final outcome seems likely to be an increase in profits.

At this point, some caveats are worth noting. First, the empirical analysis in the previous section shows that some hotel characteristics (availability of a swimming pool, animation activities and recent refurbishment) are positively associated with seasonality, and others (location in front of the beach, availability of a garden or a balcony in the room and availability of a car park) are not statistically associated with seasonality in prices. This implies that, although some characteristics are relevant components of the hotel product (see, for instance, Espinet et al, 2003; Haroutunian et al, 2005; Thrane, 2005; RigallI-Torrent and Fluvià, 2007, 2011), not all have a role in smoothing out seasonality. Another consideration is that it is possible that there is insufficient demand for an increasing supply of high-quality tourism products and destinations. In that case, reducing seasonality in prices would need to rely on increasing market shares at the expense of competitors. Besides, we are assuming no changes in the competitive strategies of rival firms and destinations. It is possible that competitors respond to changes in the competitive strategy of their rivals (especially when that change may imply a loss in market share). If rival firms counter by increasing quality and/or reducing price, then profits may fall as firms compete for a share of a pie of given size.

\section{Destination characteristics and seasonality}

The findings with respect to the influence of different destination-specific variables on seasonality in prices are also relevant. The observation that changes in demand related to climatic conditions constitute one of the factors explaining seasonality in prices (and in the number of visitors) may seem obvious. Nevertheless, according to the framework of analysis established above, a priori this could not be taken for granted. It is a hypothesis that needed to be contrasted, since lower seasonality in the number of visitors might be the result of firms lowering their prices in the off-peak season. Besides the effects of climatic conditions on price seasonality, the article provides insights into other relevant factors (after controlling for the star rating and the services offered by hotels, as well as the tour operator, accommodation type, whether the hotel is part of a chain and whether it can be classified as an apartment hotel). Indeed, Malta, the French Riviera and Naples, for instance, are Mediterranean 


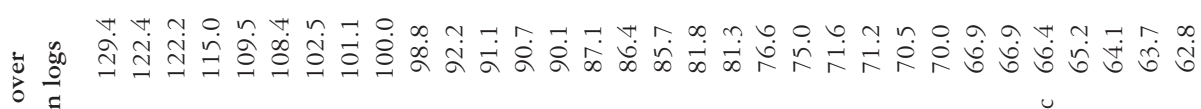

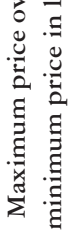

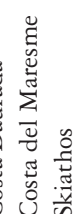

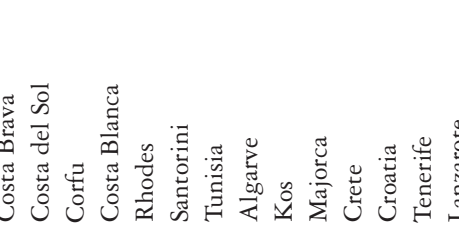

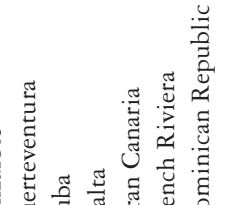

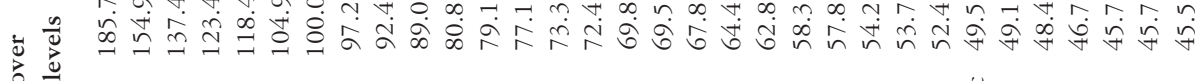

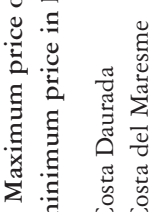

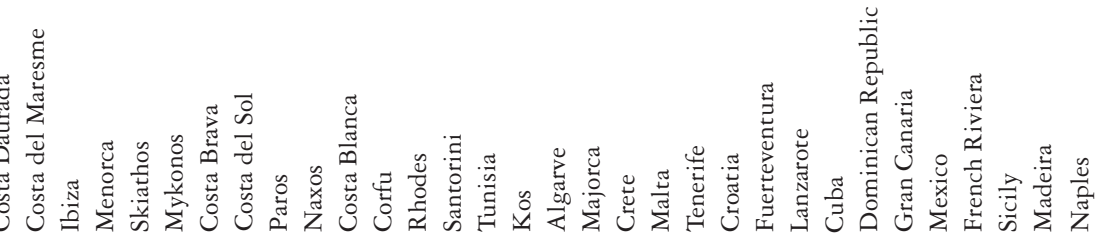

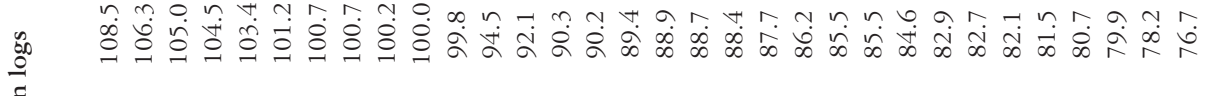

.9

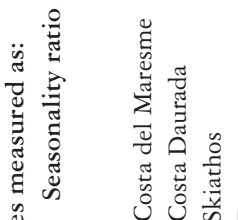

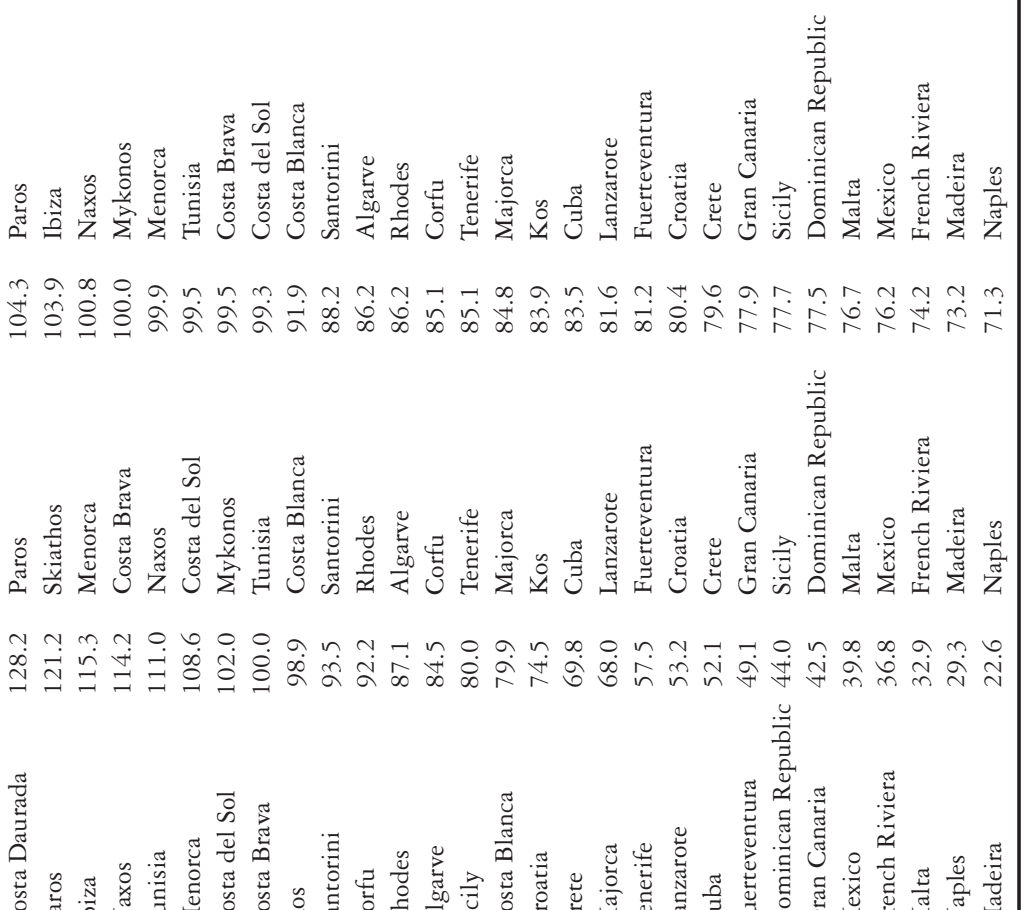

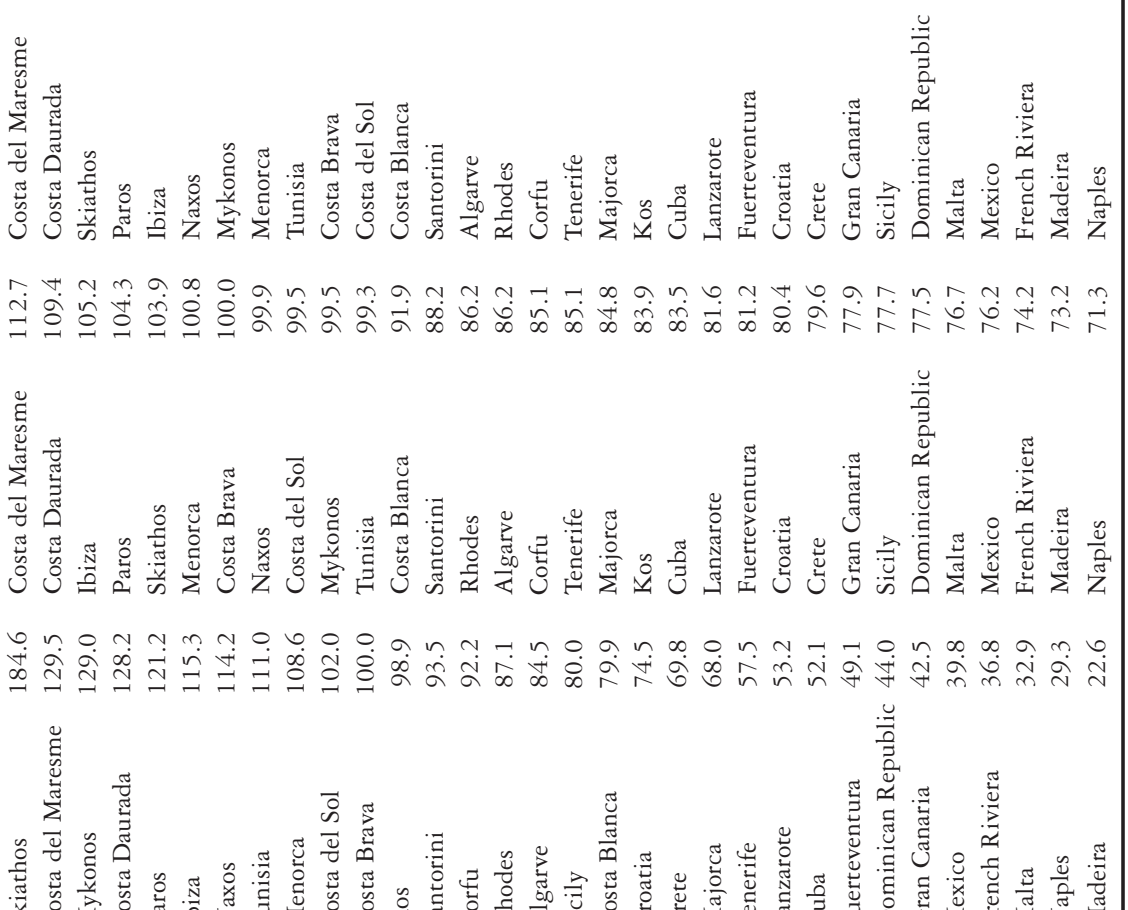

焉焉
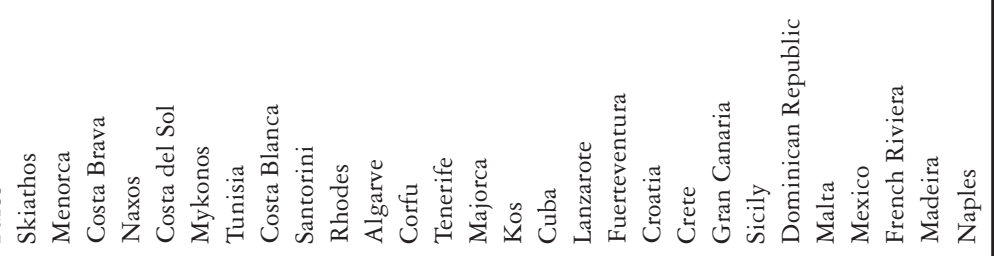

แั

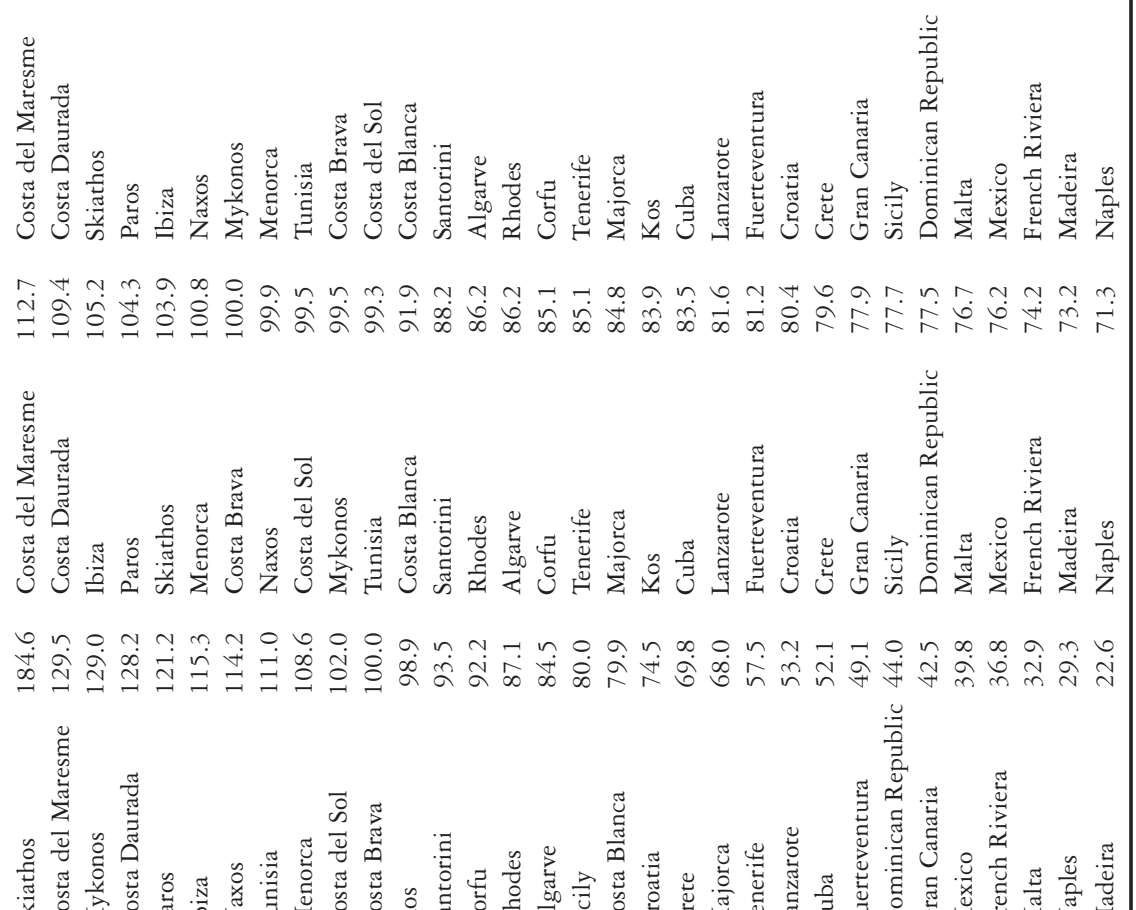

ฮี

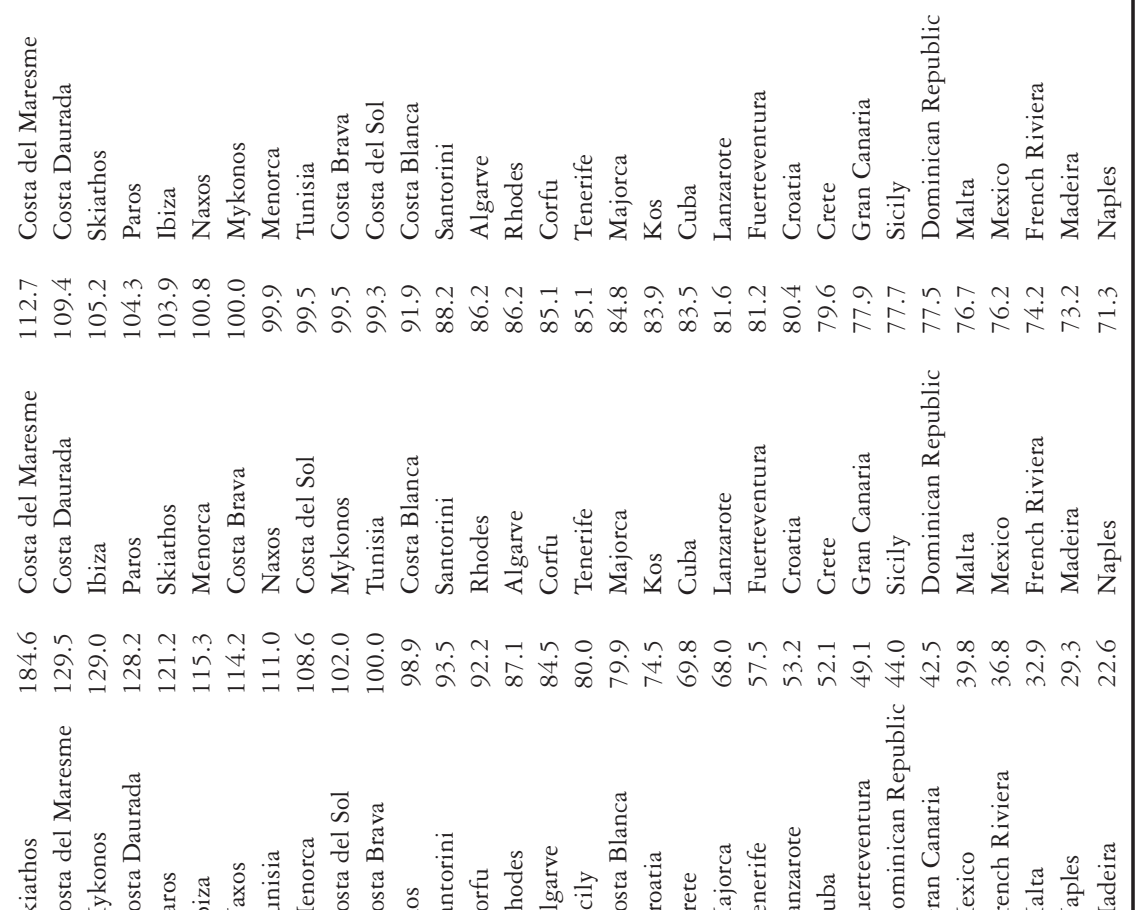

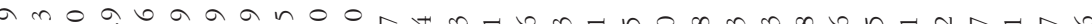

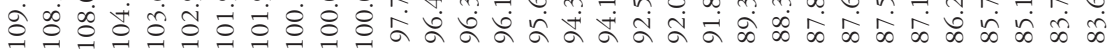

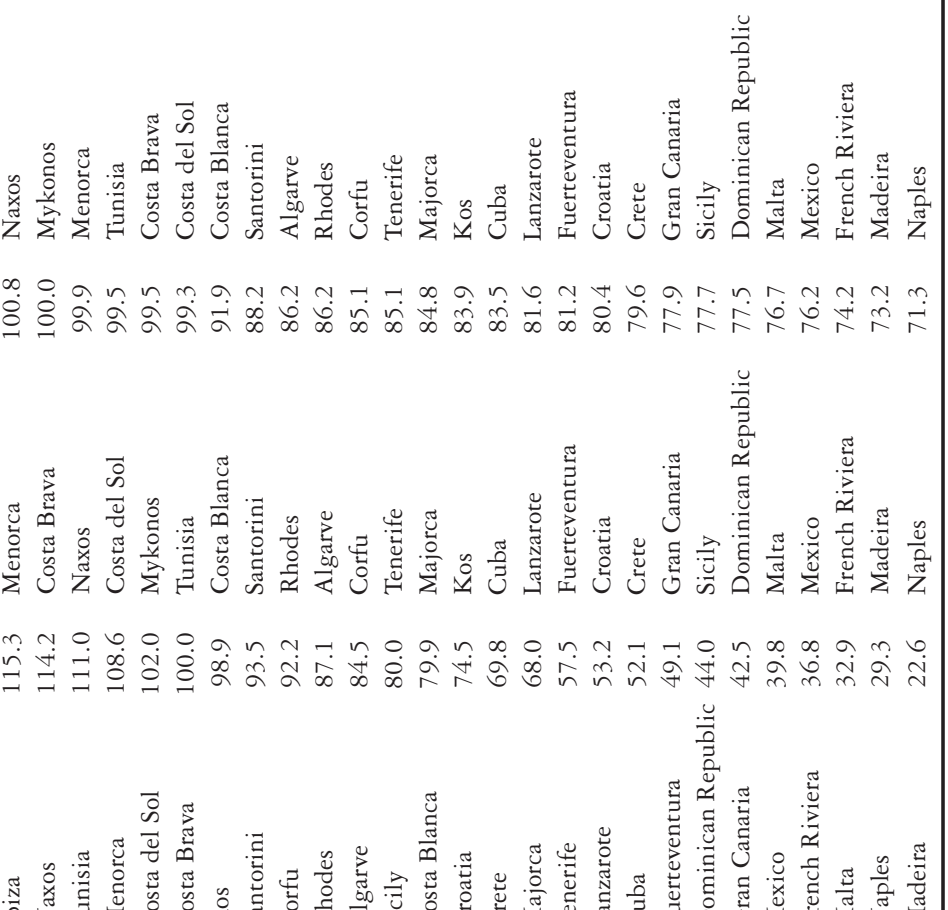

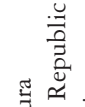

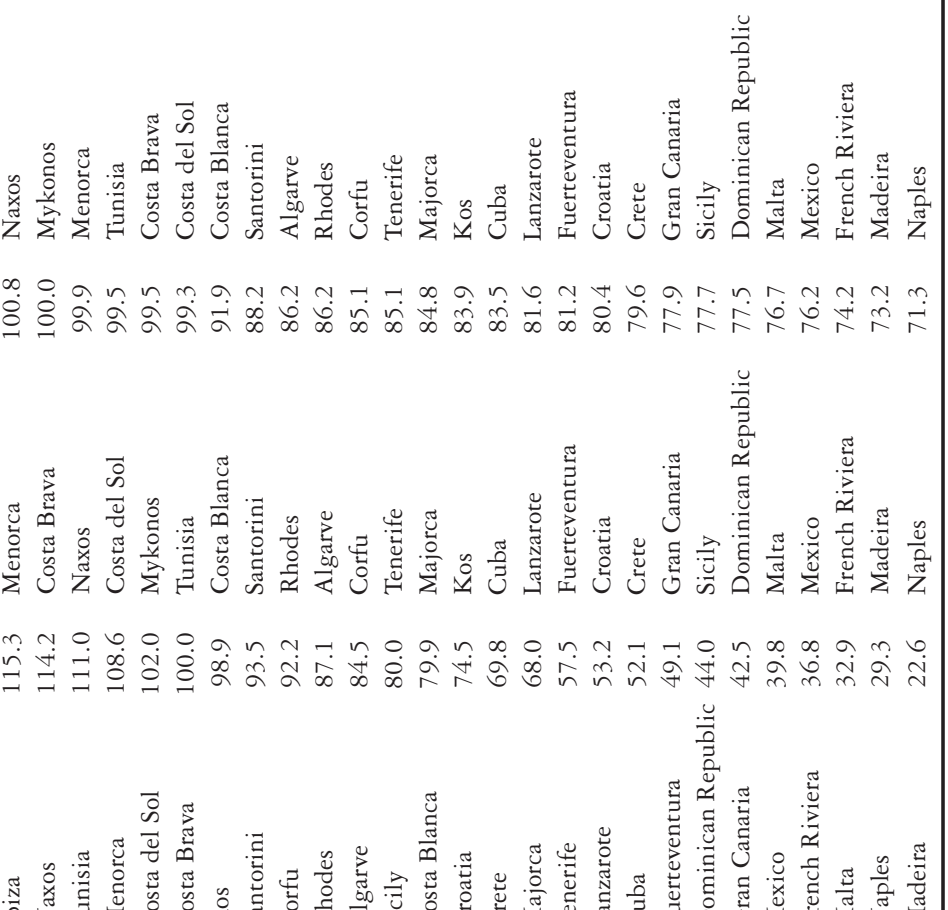

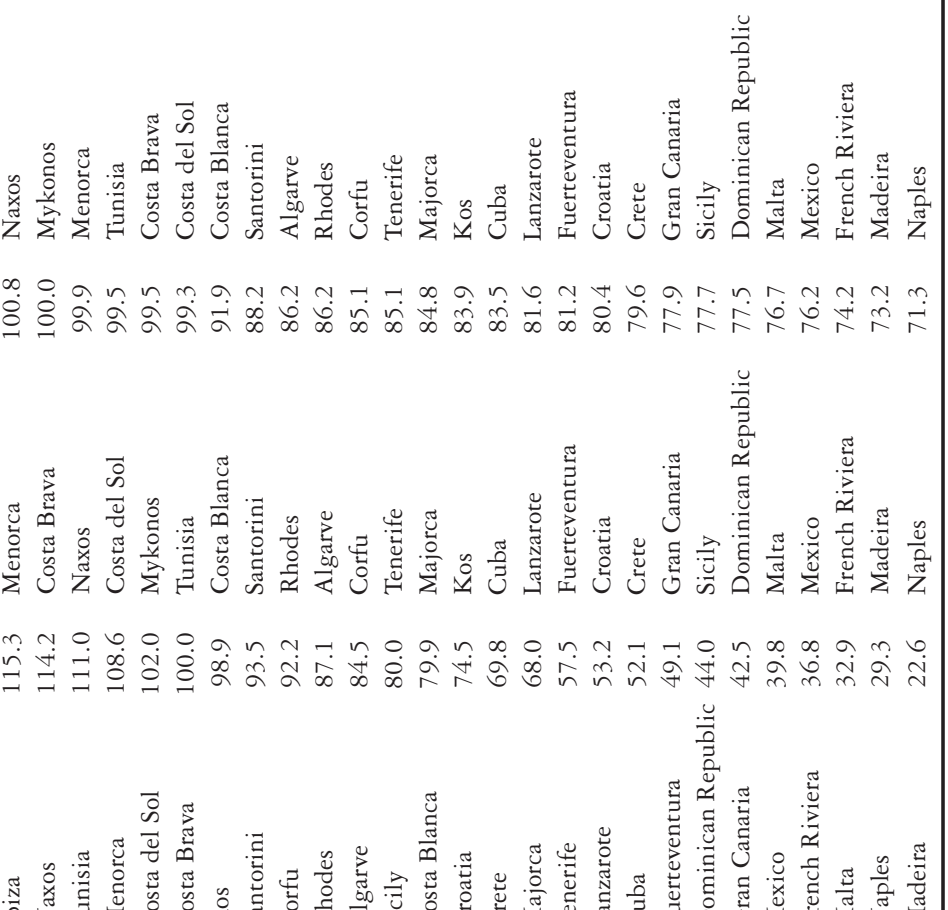


destinations showing a lower degree of seasonality than some destinations in the Caribbean, the Canary Islands or Madeira (see Table 4).

Different factors that are not explicitly included in regressions (6)-(11) lie behind those anomalies. Obvious candidates, frequently analysed in the literature, are exchange rates and transportation costs. Nevertheless, fluctuations in exchange rates do not play a role for destinations within the Euro Zone, and transportation costs do not differ much for Spanish tourists visiting Malta, the French Riviera and Naples. Since tourists' choices usually depend on the specific combination of public as well as private attributes which gives rise to the final product, other additional factors may include, for instance, brand image, exclusivity, complementary products and services (such as restaurants or museums), crowdedness, natural environment and public safety (see Rigall-ITorrent and Fluvià, 2007, 2011). All these factors are essentially related to location. Casual observation suggests that the destinations analysed in this paper differ systematically with respect to location. Obviously, this observation needs more explicit study in order to identify each specific factor and it will be the object of future research.

\section{Conclusions}

Many studies have analysed seasonality from the point of view of the number of visitors, the associated problems and the efforts of firm managers and policy makers to overcome those problems. In spite of the profusion of research on seasonality, there are no studies analysing seasonality in prices. This paper, which represents a first step in filling this void, considers the different supply and location factors that affect seasonality for hotels in sun-and-beach destinations from the novel perspective of prices. Analysing the seasonal patterns in prices is valuable for policy makers, managers and other stakeholders, since using prices to smooth the tourism season may be perceived by potential customers as a loss of quality and/or exclusivity. Therefore, although this practice generates higher revenues during the low tourism season, it may end up damaging the brand image of products and destinations and putting firms' future profits at risk.

By using regression analysis to examine the prices charged by 1,776 hotels in 32 sun-and-beach destinations in 11 countries, this paper finds that more hotel services and higher quality are associated with less seasonality in hotel prices. This finding (which is robust to different measures of seasonality in prices) immediately suggests that increasing the quality of hotel accommodation may be a promising way to reduce seasonality in prices. Nevertheless, the paper's analysis finds that other factors are also relevant to seasonality in prices. One of these is related to climatic conditions: the paper provides evidence that proximity to the equator seems to be a factor that diminishes seasonality in prices. Although climatic conditions are likely to explain some of the observed seasonality in prices, the results also strongly suggest that other factors (apart from exchange rates and transportation costs) linked to the different destinations where hotels are located are relevant when explaining seasonal variability. Further research is necessary to identify these additional factors. Nevertheless, this paper's results and previous research suggest that it is 
reasonable to assume that there is a significant link between prices, exclusivity, brand image and seasonality.

\section{References}

Aguiló, P.M., Alegre, J., and Riera, A. (2001), 'Determinants of the price of German tourist packages on the Island of Mallorca', Tourism Economics, Vol 7, No 1, pp 59-74.

Balsells, F. (2010), '5.000 universitarios británicos toman Salou en busca de alcohol barato y sexo fácil' [5,000 British university students invade Salou in search of cheap alcohol and easy sex], El País, $30 \mathrm{March}$, www.elpais.com/articulo/cataluna/5000/universitarios/britanicos/toman/Salou/ busca/alcohol/barato/sexo/facil/elpepiespcat/20100330elpcat_6/Tes, accessed 29 December 2010.

Baum, T., and Lundtorp, S. (2001), 'Seasonality in tourism: an introduction', in Baum, T., and Lundtorp, S., eds, Seasonality in Tourism, Elsevier Science, Oxford, pp 1-4.

Butler, R.W. (2001), 'Seasonality in tourism: issues and implications', in Baum, T., and Lundtorp, S., eds, Seasonality in Tourism, Elsevier Science, Oxford, pp 5-21.

Capó Parrilla, J., Riera Font, A., and Rosselló Nadal, J. (2007), 'Accomodation determinants of seasonal patterns', Annals of Tourism Research, Vol 34, No 2, pp 422-436.

Caves, R.E., and Greene, D.P. (1996), 'Brands' quality levels, prices, and advertising outlays: empirical evidence on signals and information costs', International Journal of Industrial Organization, Vol 14, No 1, pp 29-52.

Chung, J.Y. (2009), 'Seasonality in tourism: a review', e-Review of Tourism Research, Vol 7, No 5, pp 82-96.

Commons, J., and Page, S. (2001), 'Managing seasonality in peripheral tourism regions: the case of Northland, New Zealand', in Baum, T., and Lundtorp, S., eds, Seasonality in Tourism, Elsevier Science, Oxford, pp 153-172.

Cox, L.J., and Vieth, G.R. (2003), 'Hotel investment in open area', Annals of Tourism Research, Vol 30, No 2, pp 342-352.

Crouch, G.I. (1994), 'A meta-analysis of tourism demand', Annals of Tourism Research, Vol 22, No 1, pp 103-118.

Cuñado, J., Gil-Alana, L.A., and Pérez de Gracia, F. (2005), 'The nature of seasonality in Spanish tourism time series', Tourism Economics, Vol 11, No 4, pp 483-499.

Espinet, J.M., Saez, M., Coenders, G., and Fluvià, M. (2003), 'Effect on prices of the attributes of holiday hotels: a hedonic prices approach', Tourism Economics, Vol 9, No 2, pp 165-177.

Falk, M. (2008), 'A hedonic price model for ski lift tickets', Tourism Management, Vol 29, No 6, pp 1172-1184.

Fernández-Morales, A., and Mayorga-Toledano, M.C. (2008), 'Seasonal concentration of the hotel demand in Costa del Sol: a decomposition by nationalities', Tourism Management, Vol 29, No 5 , pp 940-949.

Flognfeldt, T. (2001), 'Long-term positive adjustments to seasonality: consequences of summer tourism in the Jotunheimen area, Norway', in Baum, T., and Lundtorp, S., eds, Seasonality in Tourism, Elsevier Science, Oxford, pp 109-117.

Gil-Alana, L.A., Pérez de Gracia, F., and Cuñado, J. (2004), 'Seasonal fractional integration in the Spanish Tourism Quarterly Time Series', Journal of Travel Research, Vol 42, No 4, pp 408-414.

Goh, C., and Law, R. (2002), 'Modeling and forecasting tourism demand for arrivals with stochastic nonstationary seasonality and intervention', Tourism Management, Vol 23, No 5, pp 499-510.

Haroutunian, S., Mitsis, P., and Pashardes, P. (2005), 'Using brochure information for the hedonic analysis of holiday packages', Tourism Economics, Vol 11, No 1, pp 69-84.

Hartman, R.S. (1989), 'Hedonic methods for evaluating product design and pricing strategies', Journal of Economics and Business, Vol 41, No 3, pp 197-212.

Hjorth-Andersen, C. (1991), 'Quality indicators: in theory and in fact', European Economic Review, Vol 35, No 8, pp 1491-1505.

Jang, S. (2004), 'Mitigating tourism seasonality: a quantitative approach', Annals of Tourism Research, Vol 31, No 4, pp 819-836.

Jeffrey, D., and Barden, R.R.D. (2001), 'An analysis of the nature, causes and marketing implications of seasonality in the occupancy performance of English hotels', in Baum, T., and Lundtorp, S., eds, Seasonality in Tourism, Elsevier Science, Oxford, pp 119-140.

Jones, P., and Hudson, J. (1996), 'Signalling product quality: when is price relevant?', Journal of Economic Behavior and Organization, Vol 30, No 2, pp 257-266. 
Kennedy, E., and Deegan, J. (2001), 'Seasonality in Irish tourism, 1973-1995', in Baum, T., and Lundtorp, S., eds, Seasonality in Tourism, Elsevier Science, Oxford, pp 51-74.

Kimes, S.E. (2000), 'A strategic approach to yield management', in Ingold, A., McMahon-Beattie, U., and Yeoman, I., eds, Yield Management: Strategies for the Service Industries, Thomson, London, pp 3-14.

Klemm, M., and Rawel, J. (2001), 'Extending the school holiday season: the case of Eurocamp', in Baum, T., and Lundtorp, S., eds, Seasonality in Tourism, Elsevier Science, Oxford, pp 141-151.

Koenig, N., and Bischoff, E.E. (2003), 'Seasonality of tourism in Wales: a comparative analysis', Tourism Economics, Vol 9, No 3, pp 229-254.

Krakover, S. (2000), 'Partitioning seasonal employment in the hospitality industry', Tourism Management, Vol 21, No 5, pp 461-471.

Kulendran, N., and Wong, K.K.F. (2005), 'Modeling seasonality in tourism forecasting', Journal of Travel Research, Vol 44, No 2, pp 163-170.

Lim, C., and McAleer, M. (2001), 'Monthly seasonal variations: Asian tourism to Australia', Annals of Tourism Research, Vol 28, No 1, pp 68-82.

Lundtorp, S. (2001), 'Measuring tourism seasonality', in Baum, T., and Lundtorp, S., eds, Seasonality in Tourism, Pergamon, Oxford, pp 23-50.

Lundtorp, S., Rassing, C.R., and Wanhill, S. (2001), 'Off-season is no season: the case of Bornholm', in Baum, T., and Lundtorp, S., eds, Seasonality in Tourism, Elsevier Science, Oxford, pp 89-108.

Martin, A. (2010),'Shame in Spain! British student hordes cause outrage on five-day drink rampage through resort', Daily Mail, 2 April, www.dailymail.co.uk/news/worldnews/article-1262543/Fivethousand-British-students-spark-outrage-drunken-spring-break-Spanish-seaside-resort.html, accessed 29 December 2010.

Milgrom, P., and Roberts, J. (1986), 'Price and advertising signals of product quality', Journal of Political Economy, Vol 94, No 4, pp 796-821.

Monty, B., and Skidmore, M. (2003), 'Hedonic pricing and willingness to pay for bed and breakfast amenities in Southeast Wisconsin', Journal of Travel Research, Vol 42, No 2, pp 195-199.

Papatheodorou, A. (2002), 'Exploring competitiveness in Mediterranean resorts', Tourism Economics, Vol 8, No 2, pp 133-150.

Phillips, R.L. (2005), Pricing and Revenue Optimization, Stanford University Press, Stanford, CA.

Rigall-I-Torrent, R., and Fluvià, M. (2007), 'Public goods in tourism municipalities: formal analysis, empirical evidence and implications for sustainable development', Tourism Economics, Vol 13, No 3, pp 361-378.

Rigall-I-Torrent, R., and Fluvià, M. (2011), 'Managing tourism products and destinations embedding public good components: a hedonic approach', Tourism Management, Vol 32, No 2, pp 244 255.

Rosselló Nadal, J., Riera Font, A., and Sansó Rosselló, A. (2004), 'The economic determinants of seasonal patterns', Annals of Tourism Research, Vol 31, No 3, pp 697-711.

Shy, O. (2008), How to Price: A Guide to Pricing Techniques and Yield Management, Cambridge University Press, New York.

Sorensen, N.K. (2001), 'Modelling the seasonality of hotel nights in Denmark by county and nationality', in Baum, T., and Lundtorp, S., eds, Seasonality in Tourism, Elsevier Science, Oxford, pp 75-88.

Talluri, K.T., and van Ryzin, G.J. (2004), The Theory and Practice of Revenue Management, Kluwer Academic, Boston, Dordrecht, and London.

Thrane, C. (2005), 'Hedonic price models and sun-and-beach package tours: the Norwegian case', Journal of Travel Research, Vol 43, No 3, pp 302-308.

White, H. (1980), 'A heteroskedasticity-consistent covariance matrix estimator and a direct test for heteroskedasticity', Econometrica, Vol 48, No 4, pp 817-838.

Wolinsky, A. (1983), 'Prices as signals of product quality', Review of Economic Studies, Vol 50, No 4, pp 647-658. 